\title{
Adverse Effects of Permanent Waving and Hair Relaxation-Assessment by Scanning Electron Microscopy (SEM)
}

\author{
Tokuya Omi ${ }^{1,2 *}$, Seiji Kawana ${ }^{2}$ \\ ${ }^{1}$ Department of Dermatology, Queen's Square Medical Center, Yokohama, Japan; ${ }^{2}$ Department of Dermatology, Nippon Medical \\ School, Tokyo, Japan. \\ Email: ${ }^{*}$ t.omi@queens-sq.or.jp
}

Received July $17^{\text {th }}, 2013$; revised August $16^{\text {th }}, 2013$; accepted August $23^{\text {rd }}, 2013$

Copyright (C) 2013 Tokuya Omi, Seiji Kawana. This is an open access article distributed under the Creative Commons Attribution License, which permits unrestricted use, distribution, and reproduction in any medium, provided the original work is properly cited.

\begin{abstract}
Permanent waving is very popular in Japan. Polypeptide chains (main chains) form the principal components of hair, and they are lined up longitudinally. Hair relaxation is also called straight permanent waving, and there are methods that change curly or wavy hair into straight hair. Hair damage as a result of winding, combing, and using high-temperature hairdressing irons is also often seen. By using scanning electron micrographs (SEM) we showed broken hairs and hair damage caused by permanent wave solutions. The hair damage is obvious when comparisons are made with the condition of the hair surface, condition of the cuticle, etc. Hair swelling by permanent wave solutions, manipulations such as winding, etc., inadequate rinsing with water, procedures on injured hair at the outset, etc., are considered possible reasons for any of these types of injury.
\end{abstract}

Keywords: Permanent Waving; Hair Relaxation; Scanning Electron Micrographs (SEM); Hair Damage

\section{Introduction}

Permanent waving and hair relaxation are techniques that are widely performed in Japan, and they are very popular. The mechanisms by which they are performed are oxidation and reduction reactions that are achieved by using permanent wave solutions. Injuries caused by permanent wave solutions that have been reported thus far have been primarily irritant contact dermatitis as a result of the oxidizing and reducing actions of permanent wave solutions, and in some cases there has been allergic contact dermatitis. There have also been hand injuries suffered by beauticians. In this paper we outline the principles of permanent waving and hair relaxation, and we discuss broken hair and hair damage by permanent wave solutions, using scanning electron micrographs.

\section{Discussion}

\subsection{The Principle of Permanent Waving [1-4]}

Polypeptide chains (main chains) form the principal components of hair, and they are lined up longitudinally. Ad-

\footnotetext{
${ }^{*}$ Corresponding author.
}

jacent main chains are connected by cysteine chains by cysteine bonds, electrical salt bonds, hydrogen bonds, etc., in the form of side chains, and they form a network. The springiness and resilience of hair are achieved by these side chains.

Permanent wave solutions have two components: solution 1 and solution 2. After using solution 1, the hair is limp instead of springy, but after using solution 2 the waves become set, and the hair's original springiness is restored. Weakening the hair's resilience by cleaving the side chains of the hair is the role of solution 1 (reduction reaction), and reconnecting the side chains in their new curved position is the role of solution 2 (oxidation reaction). Waves that have continuity are formed by this series of reactions.

Representative main ingredients of solution 1 are thioglycolic acid, cysteine, and acetylcysteine as reducing agents, and they cleave the cysteine bonds. The role of solution 2 is to reconnect the cysteine bonds through the oxidizing action of an oxidizing agent, and their active ingredients are sodium bromate, potassium bromate, and hydrogen peroxide. The solution 2 reaction is sometimes called "neutralization", but the term is used in the 
figurative sense of undoing the reaction by solution 1 , not in the chemical sense of a reaction between an acid and a base.

In addition, because the salt bonds are strongest at $\mathrm{pH}$ 4.5 - 5.5, bases such as ammonia are included in solution 1 in order to weaken these bonds, and the bonds are restored by removing the bases by rinsing with water. Because the hydrogen bonds are weakened by water, water is effective as a solvent, and the bonds are restored by drying after rinsing.

\subsection{Permanent Wave Procedure [2]}

The permanent wave procedure starts with a shampoo as pretreatment. Since the purpose of the shampoo is to allow the permanent wave solution to act evenly by removing the dust, sweat, etc., attached to the hair, using a tonic shampoo, etc., that contains scalp stimulants, etc., is inappropriate. Next, damaged hair or areas of hair where waving is not desired are protected with a creamtype hair treatment solution.

Permanent waving is not achieved simply by externally applying permanent wave solution 1 ; a step in which the hair is wound around rods according to the degree of the wave is also necessary. The rods range in diameter from $6 \mathrm{~mm}$ to $15 \mathrm{~mm}$, and winding 3 times is necessary to achieve a full wave. The choice of rods is important, because it depends on hair quality and the degree of the wave.

The preparation to use for permanent wave solution 1 reaction must be selected according to hair quality and ability to form waves. Highly alkaline preparations have stronger ability to form waves. There are two methods of applying solution 1, i.e., a water-winding method in which the solution is applied to the entire head of hair after first winding moistened scalp hair around rods, and an application-winding method in which the hair is wound around the rods after first applying the permanent waving solution to the hair. Evenly applying the permanent wave solution to all of the hair and not allowing the solution to run down onto the scalp are important precautions that are common to both methods. If the solution is allowed to remain on the face or back of the neck, it may cause contact dermatitis.

The hair is rinsed with water after completion of the solution 1 reaction. This step is essential, because many preparations that are used as solution 1 are alkaline and they weaken the oxidizing action of solution 2 .

Next, solution 2 is applied. As stated in the "principle" section above, the action of solution 2 is essential in order to maintain the wave that was produced with solution 1. Because the hair swells when it reacts with solution 1 , it has a high water content, and since, because of that, if solution 2 is applied all at once, most of it trickles down, solution 2 must be applied to the hair carefully one strand at a time.

The permanent wave is completed by treatment with solution 2. The rods are removed, and solution 2 is thoroughly rinsed away with water. The scalp as well as the hair often becomes soft and swells as a result of the oxidizing action and reducing action of the solutions, and inadequate rinsing is a possible cause of increased dandruff, etc., in patients who have received a permanent wave.

\subsection{Principle and Procedures of Relaxing Hair [5]}

Hair relaxation is also called straight permanent waving, and there are methods that change curly or wavy hair into straight hair. Commercialization of products was permitted in Japan by the Pharmaceutical Affairs Law in 1985. The principle of hair relaxation is exactly the same as the principle of permanent waving, and the sole difference is that in hair relaxation the winding around the rods in permanent waving is replaced by stretching the hair out straight with a comb. The solution 1 used for hair relaxation takes the form of a viscous cream that contains a thickener. It also has stronger reducing power than the permanent wave solution.

Combing is performed as the step that corresponds to the winding step in permanent waving. More specifically, the hair is combed out straight with a rather coarse comb. The step in which the hair is stretched out with the comb after the solution 1 reaction causes a great deal of damage to the hair, and caution is required because of the risk of damaging or breaking the hair.

In 2001, a method in which an iron is used was authorized as a substitute for combing, and a method in which hair is relaxed with a high-temperature hairdressing iron after the solution 1 reaction has become mainstream. In this method the hair is treated by placing bundles of hair, starting at the roots, between the flat plates of a straight iron heated to $180^{\circ} \mathrm{C}$ and sequentially sliding the iron to the tips of the hair.

The same as in permanent waving, the hair is then treated with solution 2 and rinsed with water.

\subsection{Safety Management and Unintended Uses of Permanent Wave Solutions}

In order to ensure the safety of permanent wave solutions, in Japan they have been designated quasi-drugs, and permission for quasi-drug production and importation is required to manufacture or import permanent wave solutions. Consequently, the administration, dosage, indications, and efficacy of permanent wave solutions have been stipulated [5].

The Japan Poison Information Center receives about 20 inquiries a year regarding incidents related to misuse of 
permanent wave solution, and close to half of the inquiries are from medical facilities [4,5]. Many of them are the result of accidental swallowing by children or eye or skin contact while using it. When solution 1 is accidentally swallowed, patients complain of abdominal symptoms because of the irritant action due to the solution's alkalinity, and when solution 2 is accidentally swallowed, they complain of abdominal symptoms 1 - 2 hours later, but inducing vomiting with solution 1 is contraindicated from the standpoint of its pharmaceutical properties.

There have also been reports of unintended uses that are inconsistent with the purposes of using permanent wave solutions. About 20 years ago, a fatal case was reported in a 6-month-old infant in addition to acute dermatitis medicamentosa in a group of beauticians who attempted to popularize an acidic permanent wave solution as being able to improve overall health and having efficacy against trichophytosis and dermatitis when externally applied to the whole body. Eye injuries have also been reported as a result of permanent waving eyelashes. At both times strong notifications calling for proper use were issued by the Environmental Health Bureau of the Ministry of Health and Welfare. If by chance a solution enters the eye, solution 1 has a greater irritant action than solution 2 .

\subsection{Hair Injury by Permanent Wave Solutions}

As described above, permanent waving is performed on the basis of the oxidizing action and reducing action of permanent wave solutions [1,2], and hair damage as a result of winding, combing, and using high-temperature hairdressing irons is also often seen. Figures 1-4 are scanning electron micrographs of broken hairs and hair damage caused by permanent wave solutions. The findings in healthy hair are shown in Figure 5 for reference, and the hair damage is obvious when comparisons are made with the condition of the hair surface, condition of

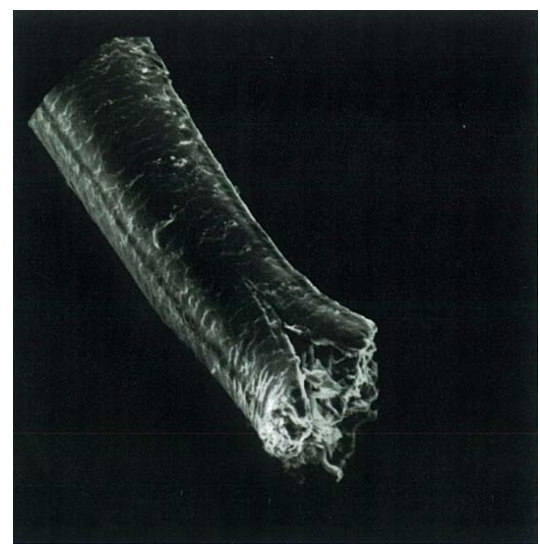

Figure 1. Broken hair as a result of a permanent wave. Broken hair is often seen during winding after the action of solution 1.

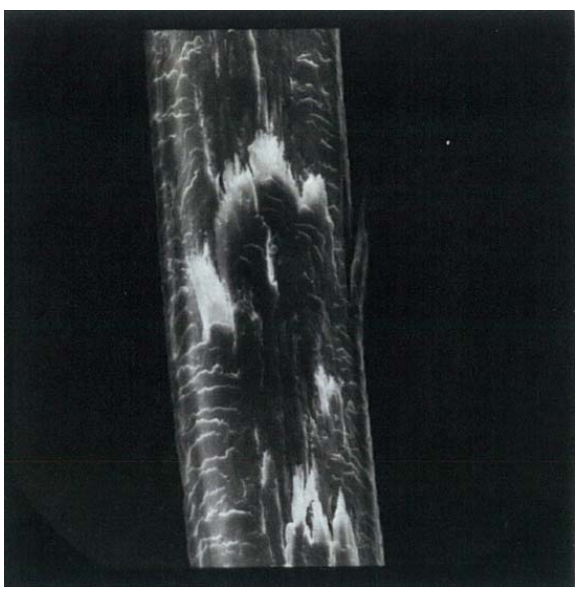

Figure 2. Damage as a result of a permanent wave. The surface of the hair is shaggy, and damage to the cuticle is also seen.

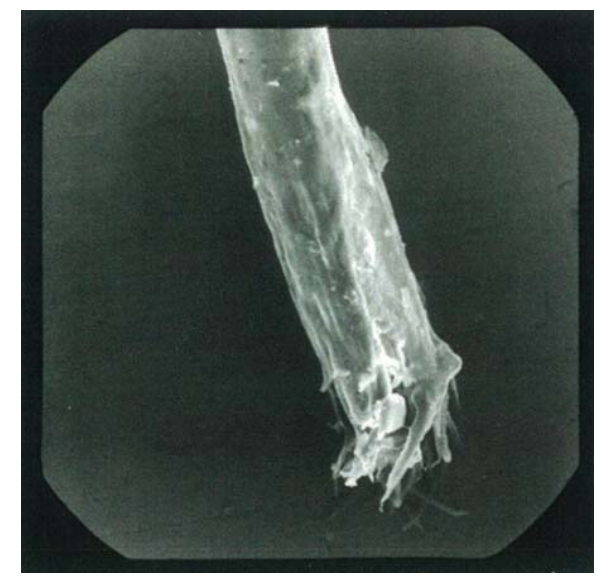

Figure 3. Broken hair as a result of hair relaxation. Hair relaxation subjects hair to considerable stress, and when solution 1 is applied close to the roots, many broken hairs like these are often seen. Combing manipulations also tend to cause hairs to break.

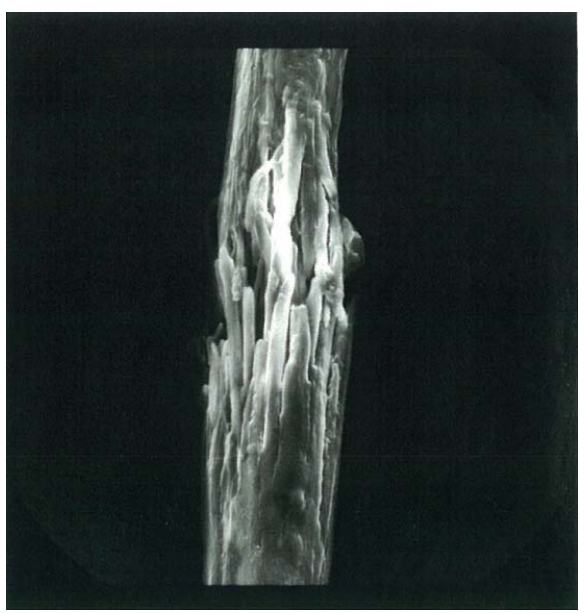

Figure 4. Damage as a result of hair relaxation. Insufficient rinsing is another possible reason besides damage due to combing or ironing after using solution 1. 


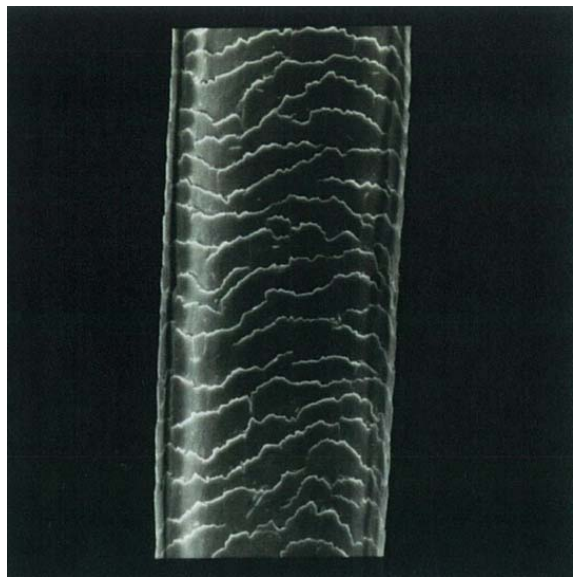

Figure 5. Healthy hair. The surface of the hair is smooth, and no damage to the cuticle is seen.

the cuticle, etc.

Hair swelling by permanent wave solutions, manipulations such as winding, etc., inadequate rinsing with water, procedures on injured hair at the outset, etc., is considered possible reasons for any of these types of injury. Using a hair dryer immediately after permanent waving is another possible cause of hair injury [6].

\subsection{Skin Injury by Permanent Wave Solutions}

Almost all of the skin injuries caused by permanent wave solutions on the basis of their oxidizing action and reducing action is primary irritant contact dermatitis, and allergic contact dermatitis is very rare [1,2].

The hairline on the forehead, behind the ears, the frontal region, etc., are the most common sites of skin injury in people who get a permanent wave, and diagnosis is easy.

By contrast, injury to the hands is very common among beauticians [7,8]. The barrier function of beauticians' skin is reduced by the mechanical irritation of giving shampoos daily and dilapidation due to using surfaceactive agents. When their hands are exposed to irritation by permanent wave solutions that have chemical actions under these circumstances, beauticians develop so-called occupational chapped hands. As a result of frequent use of cysteine permanent wave solutions in the past, a phenomenon call flaking, in which insoluble cysteine adhered and exacerbated the chapping, was also often seen, but as a result of commercialization efforts by manufacturers' based on studies of the components of permanent wave solutions, it is hardly ever seen today.

Due to similar corporate efforts, the irritant actions of permanent wave solutions have also decreased, but, need- less to say, beauticians' hand injuries are a major problem.

Furthermore, dressing up at younger ages has become more popular recently, and the number of parents who want permanent waves for their children has also increased. The cysteine content of children's hair is lower, and it is difficult to permanent wave their hair. When we also consider the irritation caused to the skin of their scalps, it appears better to avoid permanent waving children's hair as much as possible.

\section{Conclusion}

Almost all skin injury by permanent wave solutions is primarily irritant contact dermatitis due to the oxidizing action and reducing action of the permanent wave solutions. Hair damage as a result of the oxidizing action of permanent wave solution is also often seen.

\section{REFERENCES}

[1] M. O. Han, J. A. Chun, J. W. Lee and C. H. Chung, "Effects of Permanent Waving on Changes of Protein and Physicomorphological Properties in Human Hair," Journal of Cosmetic Science, Vol. 59, No. 3, 2008, pp. 203215.

[2] Japan Permanent Waving Lotion Industry Association, "Science of Wave," Revised Edition, 2002, pp. 14-19.

[3] Z. Wang, X. Ren, D. Wang, Y. Guan and L. Xeia, "Effects of Thioglycolic Acid on Parthenogenetic Activation of Xenopus Oocytes,” PLos One, Vol. 6, 2011, Article ID: e16220. doi:10.1371/journal.pone.0016220

[4] K. Suzuta, S. Ogawa, Y. Takeda and K. Kaneyama, "Intermolecular Disulfide Cross-Linked Structural Changes Induced by Permanent Wave Treatment of Human Hair with Thioglycolic Acid,” Journal of Cosmetic Science, Vol. 63, No. 2, 2012, pp. 177-196.

[5] Japan Permanent Waving Lotion Industry Association, "Science of Wave," Revised edition, 2002, pp. 36-83.

[6] Y. Lee, Y. D. Kim, H. J. Hyun, L. Pi, X. Jin and W. S. Lee, "Hair Shaft Damage from Heat and Drying Time of Hair Dryer,” Annals of Dermatology, Vol. 23, No. 4, 2011, pp. 455-462. doi:10.5021/ad.2011.23.4.455

[7] D. L. Holnes, "Recent Advances in Occupational Dermatitis," Current Opinion in Allergy and Clinical Immunology, Vol. 13, No. 2, 2013, pp. 145-150. doi:10.1097/ACI.0b013e32835e12cf

[8] P. J. Coenraads, "Hand Eczema," The New England Journal of Medicine, Vol. 367, No. 19, 2012, pp. 18291837. doi:10.1056/NEJMcp1104084 